\title{
Establishment of a rapid detection method for wheat crown rot
}

\author{
Lina Sui ${ }^{1}$, Jishun $\mathrm{Li}^{2}$, Jindong $\mathrm{Hu}^{2}$, Yan Wang ${ }^{1, *}$ \\ ${ }^{1}$ School of Biological Engineering, Qilu University of Technology, Jinan, Shandong, China \\ ${ }^{2}$ Shandong Provincial Key Laboratory of Applied Microbiology, Institute of Ecology, Shandong Academy of Sciences, Qilu University \\ of Technology (Shandong Academy of Sciences), Jinan, Shandong, China
}

\begin{abstract}
We have established a real-time fluorescent quantitative PCR system that can detect wheat crown rot rapidly and accurately quantify fungi, Fusarium species and Fusarium pseudograminearum in the rhizosphere soil of infected wheat through the standard curve produced, with a view to the early stage of wheat Provide help in predicting the occurrence of wheat crown rot.
\end{abstract}

\section{Introduction}

Fusarium spp. is a pathogen of a variety of soil-borne diseases of wheat[1], which can infect the vascular system of plants and cause the roots, stems and leaves of plants to rot. For example, Fusarium pseudograminearum is the main pathogens of wheat crown rot[2]. The infection of fusarium on wheat tissues will greatly affect the yield of wheat and the quality of wheat ears [3].

Real-time fluorescent quantitative PCR (QPCR) technology is to use fluorescence accumulation to monitor the entire process in real time by adding fluorescent groups to the DNA amplification reaction, thereby quantitatively analyzing unknown templates $[4$, 5]. This technology has been widely used in the detection of various plant pathogens after its emergence. QPCR generally has two types: SYBR Green and TaqMan probe method [4]. Both methods are involved in this experiment.

In this study, the established a rapid QPCR detection system for wheat crown rot, aiming to provide detection for the number of fungi Fusarium in rhizosphere soil, and also for the establishment of a technique for predicting the occurrence of early wheat stem rot.

\section{Materials and Methods}

\subsection{Collection and processing of soil samples}

Soil sample collection: Three plots with significant differences in the incidence of wheat crown $\operatorname{rot}(\mathrm{H})$, light (L) and non-infected plots (CK) were selected. Harvest 5 randomly selected wheat seedlings (including root system and attached rhizosphere soil) from each plot, transfer them to sterile sample bags and bring them back. Use sterile water to rinse the soil attached to the roots and then centrifuge to produce rhizosphere soil samples. Shake and combine the rhizosphere soil at each sampling point and store it at $-80^{\circ} \mathrm{C}$ before analysis.

\subsection{Preparation of fusarium spp. standard plasmid}

Take a small centrifuge tube and add $50 \mu \mathrm{L}$ of 50 $\mathrm{mmol} / \mathrm{L} \mathrm{NaOH}$ solution, pick a few hyphae with a sterile toothpick, boil water for $10 \mathrm{~min}$, add $5 \mu \mathrm{L}$ of $1 \mathrm{~mol} / \mathrm{L}$ Tris-HCl (pH 8.0) buffer, and mix well for 5000r Centrifuge for $2 \mathrm{~min}$, take the supernatant as a template.

Amplification of the target fragment. Primer sequence: Fus-F: 5'-AACTCCCAAACCCCTGTGAACATA-3'; Fus-R: 5'TTTAACGGCGTGGCCGC-3'. The primers were used to amplify Fusarium pseudograminearum. The reaction system was $25.0 \mu$ l, of which $10 \times$ PCR buffer $2.5 \mu \mathrm{l}$, dNTP $2 \mu \mathrm{l}$, Taq enzyme $0.5 \mu \mathrm{l}$, upstream and downstream primers $(10 \mu \mathrm{mol} / \mathrm{L})$ each $0.5 \mu \mathrm{l}$, template 1 . $0 \mu \mathrm{l}$, make up the volume with deionized water. The reaction conditions are: pre-denaturation at $95{ }^{\circ} \mathrm{C}$ for 2 min; denaturation at $95{ }^{\circ} \mathrm{C}$ for $45 \mathrm{~s}$, annealing at $57{ }^{\circ} \mathrm{C}$ for $30 \mathrm{~s}$, extension at $72{ }^{\circ} \mathrm{C}$ for $1.5 \mathrm{~min}, 30$ cycles; extension at $72{ }^{\circ} \mathrm{C}$ for $5 \mathrm{~min}$. The amplified fragment was ligated with the cloning vector pMD-18T, Top10 competent cells were transformed, plated (containing ampicillin resistance), and sequenced.

Preparation of standard products: Extract the positive clone plasmid as the QPCR reaction standard product. The concentration of the dilution standard was $\left(1 \times 10^{-}\right.$ ${ }^{6} \mathrm{ng} \sim 1 \mathrm{ng}$ ) seven different gradients, and $\mathrm{ddH} 2 \mathrm{O}$ was used as the negative control.

\subsection{Preparation of fungal standard plasmid}

Amplification of the target fragment .Primer sequence: ITS-F(5'-3'): CTTGGTCATTTAGAGGAAGTAA; ITS$\mathrm{R}\left(5^{\prime}-3\right)$ : GCTGCGTTCTTCATCGATGC. Use this

\footnotetext{
*Corresponding author: wangyan20210312@163.com

First author: 17862979276@163.com
} 
primer to amplify the plasmid, and the reaction system and sequence determination are the same as those of Fusarium.

Preparation of standard plasmid: the same as the construction method of Fusarium

\subsection{Preparation of F.pseudograminearum standard plasmid}

Amplification of the target fragment .Primer sequence: FP-F(5'-3'): ATCATTCGAATCGCTCGACG; FP-R(5'-

3): AAAAATTACGACAAAGCCGTAAAAA. FP-P(5'3): ACTCGACACGCGCCTGTTACCC.Use this primer to amplify the plasmid, and the reaction system and sequence determination are the same as those of Fusarium.

Preparation of standard plasmid: the same as the construction method of Fusarium.

\subsection{Establishment of real-time QPCR detection system for fusarium spp.}

Standard preparation: The diluted standard plasmid is $\left(1 \times 10^{-6} \mathrm{ng}-1 \mathrm{ng}\right)$ seven different gradients, and $\mathrm{ddH} 2 \mathrm{O}$ is used as a negative control. The reaction system was 20.0 $\mu$, including $2 \times$ Universal SYBR Green Fast qPCR mix 10. $0 \mu \mathrm{l}$, upstream and downstream primers $(10 \mu \mathrm{mol} / \mathrm{L})$ each $1.0 \mu \mathrm{l}$, template $1.0 \mu \mathrm{l}$, and deionized water $7.0 \mu \mathrm{l}$. Reaction conditions: $95{ }^{\circ} \mathrm{C}$ pre-denaturation for $2 \mathrm{~min}$; $95{ }^{\circ} \mathrm{C}$ denaturation for $45 \mathrm{~s}, 51{ }^{\circ} \mathrm{C}$ annealing for $30 \mathrm{~s}$, $72{ }^{\circ} \mathrm{C}$ extension for $1 \mathrm{~min}, 72{ }^{\circ} \mathrm{C}$ final extension for 7 min, 39 cycles. The melting curve temperature setting range is $60 \sim 95.0{ }^{\circ} \mathrm{C}$. Amplification curve, standard curve are automatically drawn by Bio-rad fluorescent quantitative PCR instrument.

\subsection{Establishment of real-time QPCR detection system for F.pseudograminearum}

The reaction system is the same as that of Fusarium. PCR conditions were $95^{\circ} \mathrm{C} 10 \mathrm{~min}, 95^{\circ} \mathrm{C} 30 \mathrm{~s}, 51^{\circ} \mathrm{C} 30$ $\mathrm{s}, 72{ }^{\circ} \mathrm{C}$ extension $1 \mathrm{~min}, 72^{\circ} \mathrm{C}$ final extension $7 \mathrm{~min}, 39$ cycles.

\subsection{Establishment of real-time QPCR detection system for fungi}

F.pseudograminearum reaction system $(20 \mu \mathrm{l})$ : including $10 \mu \mathrm{L} 2 \times$ TaqMan Fast qPCR Master Mix, 10 $\mu \mathrm{M}$ TaqMan probe $0.3 \mu \mathrm{L}, 10 \mu \mathrm{M}$ forward and reverse primers each $0.5 \mu \mathrm{L}$, DNF Buffer $2 \mu \mathrm{L}, 1 \mu \mathrm{L}$ DNA standard Sample template, ddH2O fills up $20 \mu \mathrm{L}$. Reaction conditions: 3 minutes at $95^{\circ} \mathrm{C}, 15$ seconds at $94^{\circ} \mathrm{C}, 30$ seconds at $60^{\circ} \mathrm{C}$, a total of 40 cycles of reaction.

\subsection{Application of fluorescence quantitative PCR in wheat rhizosphere soil}

Extraction of soil genome: Take $0.3 \mathrm{~g}$ fresh weight of each soil, and extract the genome with the DNeasy®PowerSoil®Kit.
Gene copy number calculation formula:

$($ copies $/ g)=S Q \times 6.02 \times 1023 \times 10-9 \times 100 /(D N A$ length $\times 660 \times 0.3$ )

where SQ is the result of fluorescence quantitative PCR and DNA length is the standard the size of the plasmid(fungal plasmid is $3171 \mathrm{bp}$,Fusarium spp. 3135 bp and F.pseudograminearum 2776bp), 100 is the volume of DNA extracted from the soil sample (uL), and $0.3 \mathrm{~g}$ is the mass of the soil sample.

\section{Results \& Discussion}

\subsection{Gel electrophoresis PCR results}

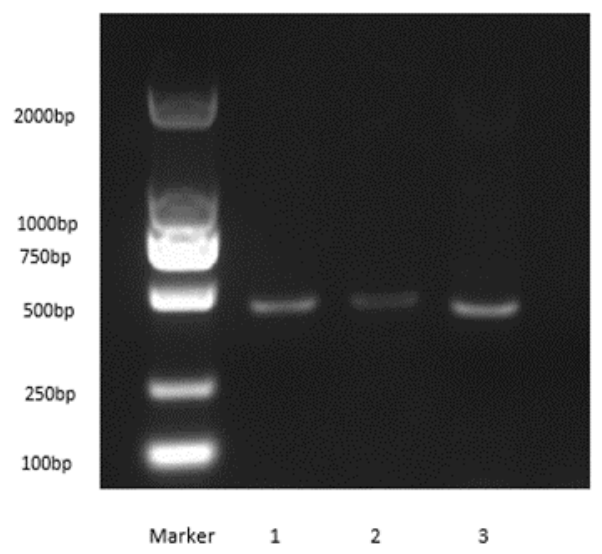

Figure 1 PCR gel electrophoresis of fungal fragments

Note: Marker DL2000, lanes 1, 2, 3 are fragments amplified by fungal primers.

The result is shown in Figure 1. The fragment size is $479 \mathrm{bp}$, and the PCR product size is correct. The amplified band is clearer, and there is no primer dimer influence.

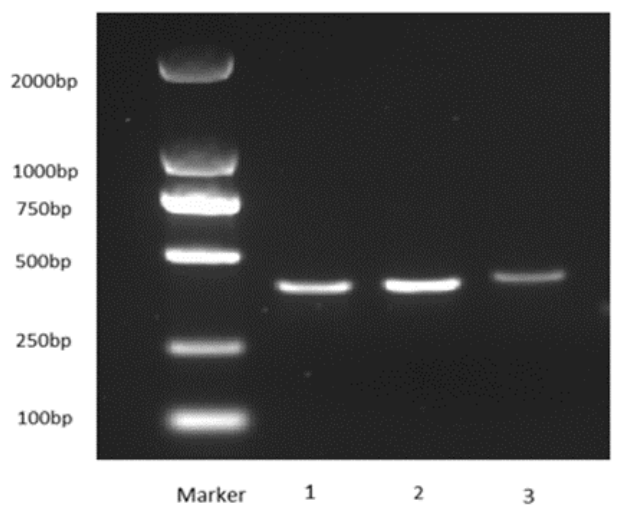

Figure 2 PCR gel electrophoresis of Fusarium spp.

Note: Marker DL2000, lanes 1, 2, 3 are the fragments amplified by the universal primers for Fusarium

The result is shown in Figure 2. The fragment size is $443 \mathrm{bp}$, the PCR product size is correct 


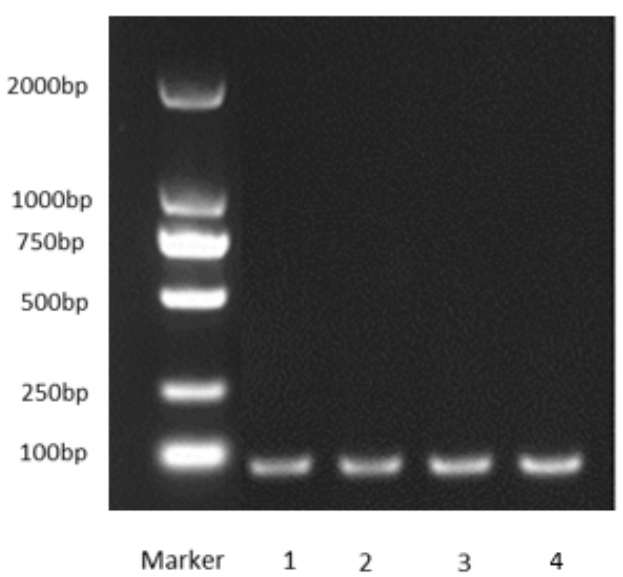

Figure 3 PCR gel electrophoresis of Fusarium pseudograminearum

Note: Marker DL2000, lanes 1, 2, 3,4 are the fragments amplified for $F$. pseudograminearum. The result is shown in Figure 3. The fragment size is $84 \mathrm{bp}$, the PCR product size is correct.

\subsection{Fungi standard curve}
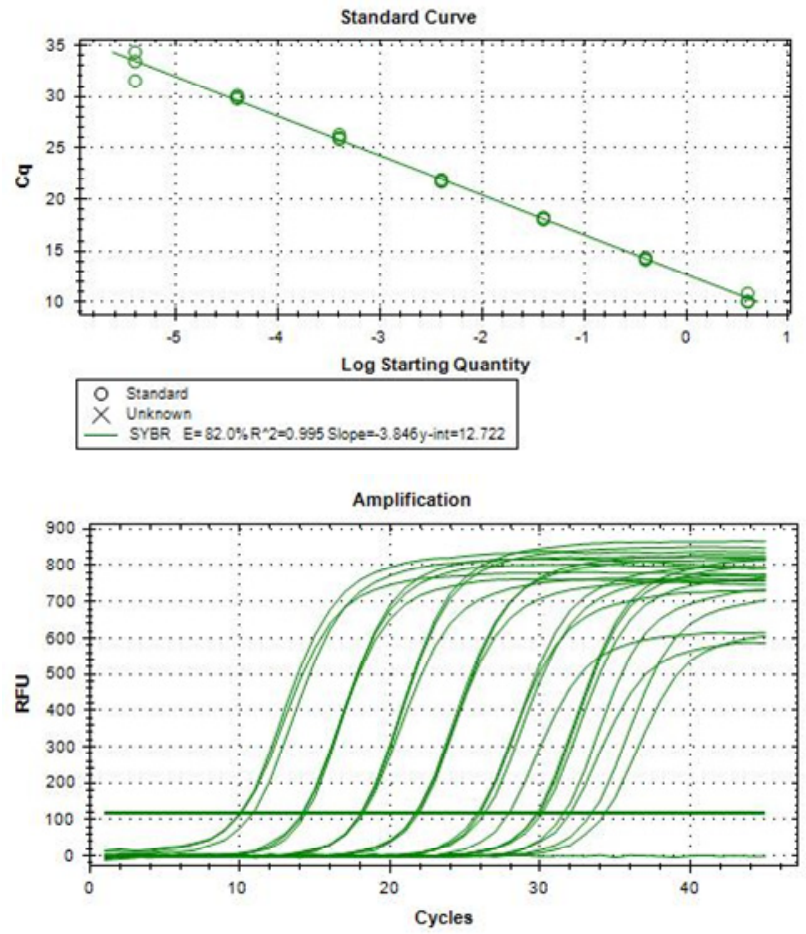

Figure 4 Fungal fluorescence quantitative PCR standard curve and amplification curve

The results of the fungal standard curve are shown in Figure 4. The amplification curves of the 7 plasmid standards are relatively smooth, and each cycle threshold is evenly spaced. The linear relationship is good, and the sensitivity can reach $1 \mathrm{fg}$.

Comprehensive primer PCR gel electrophoresis detection and QPCR system results can show that the established fungal QPCR system and reaction conditions meet our needs for accurate quantification of fungi.curve and melting curve are automatically drawn by Bio-rad fluorescent quantitative PCR instrument.

\subsection{Fusarium standard curve}
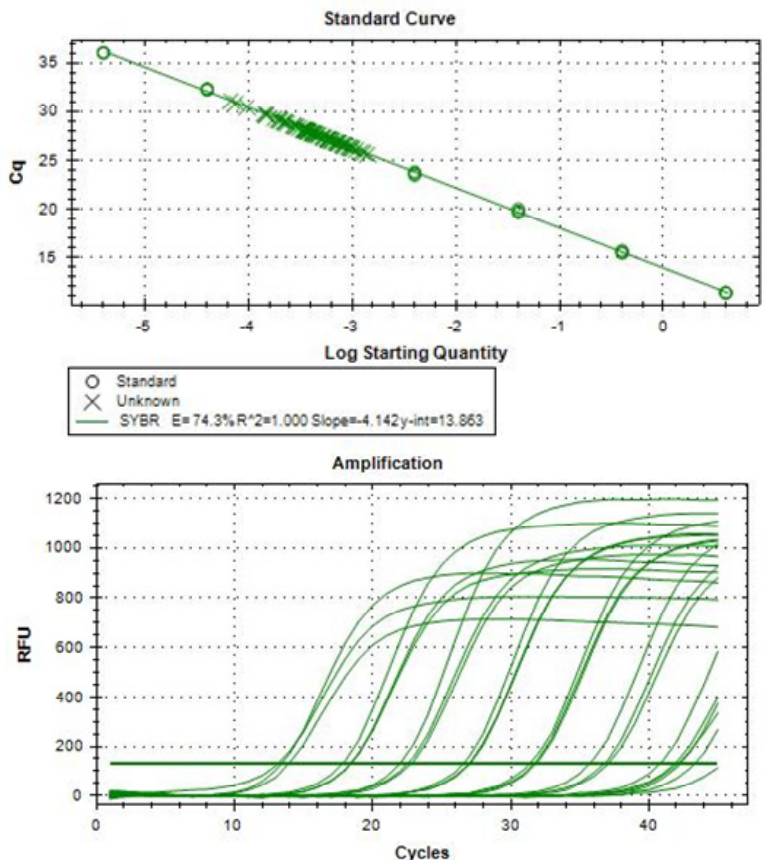

Figure 5 Standard curve and amplification curve of Fusarium by fluorescence quantitative PCR

The results of the standard curve of Fusarium are shown in Figure 5. The 7 gradients of standard products have a good linear relationship, and the sensitivity can reach $1 \mathrm{fg}$. The amplification curve is smooth, typical s-shape. Combined with the above results, it can be shown that the established QPCR detection system for Fusarium in wheat rhizosphere soil is reasonable and sensitive, and can meet our requirements for accurate quantification of Fusarium.

\subsection{Fusarium pseudograminearum standard curve}
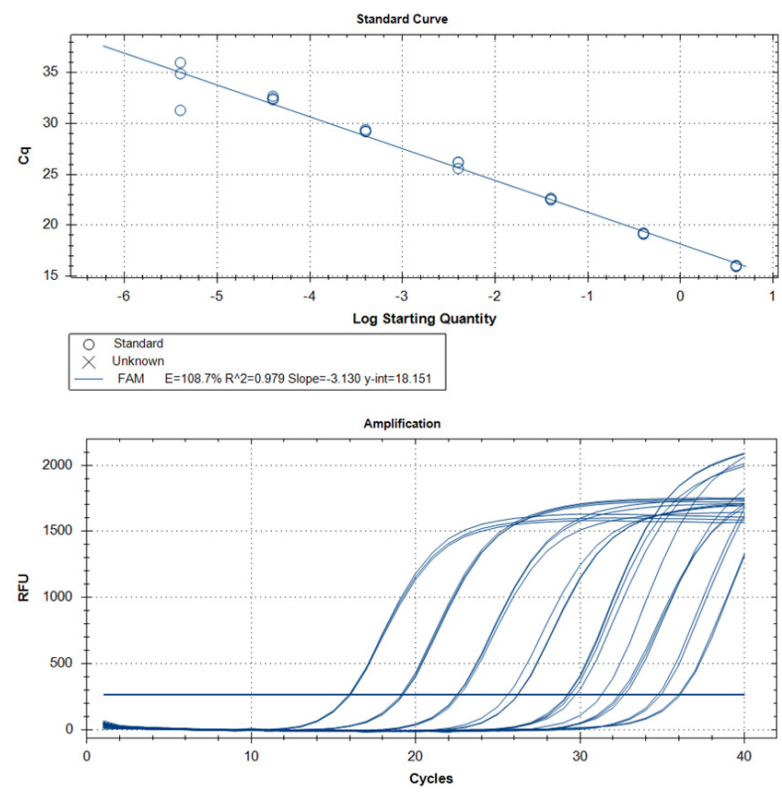

Figure 6 Standard curve, melting curve and amplification curve of Fusarium by fluorescence quantitative PCR 
The results of the standard curve of $F$. pseudograminearum are shown in Figure 6. The 7 gradients of standard products have a good linear relationship, and the sensitivity can reach $1 \mathrm{fg}$. Combined with the above results, it can be shown that the established QPCR detection system for $F$. pseudograminearum in wheat rhizosphere soil is reasonable and sensitive, and can meet our requirements for accurate quantification of Fusarium.

\subsection{Application of Real-time QPCR Detection System}

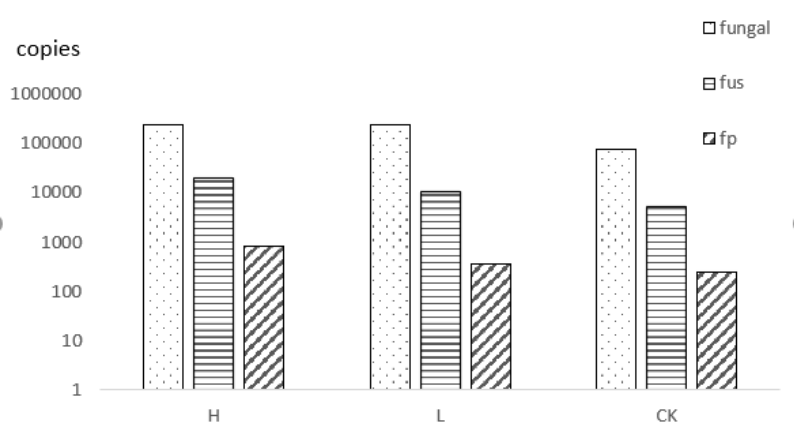

Figure 7 Copy numbers of fungi and Fusarium in wheat rhizosphere soil of different plots

The results are shown in Figure 7. The more severe the disease, the more fungi, Fusarium and Fusarium pseudograminearum, and the degree of disease is positively correlated with the amount of Fusarium. The result is consistent with the condition of wheat.

\section{Conclusions}

In this study, the primers used in the fluorescence quantitative PCR reaction, the amplification curve obtained from the reaction, the melting curve and the standard curve all reached the ideal conditions for the fluorescence quantitative reaction. The QPCR detection system for fungi, Fusarium and F.pseudograminearum established in the experiment can accurately quantify the content of fungi and Fusarium in wheat rhizosphere soil and can predict the occurrence of early wheat crown rot . The fluorescence quantitative detection method established in this experiment is more accurate than the traditional plate dilution method for the quantification of fungi and Fusarium in the soil. The copy number of fungi and Fusarium in wheat rhizosphere soil can be obtained through the established QPCR reaction system. Through the establishment of F.pseudograminearum quantitative PCR, we can predict the early incidence of wheat stem rot. It can be seen from the results that the amount of fungi and Fusarium in the rhizosphere soil in areas with severe disease is much higher than that in areas with mild or no disease, indicating that the degree of disease is positively correlated with the amount of fungi and Fusarium in the soil . There are also significant differences in the content of Fusarium pseudograminearum. The establishment of this method can provide help for predicting the occurrence of early wheat crown rot.

\section{Acknowledgments}

This research was supported by the Shandong Province Major Science and Technology Innovation Project (2019JZZY010718)

\section{References}

1.Bentley AR, Tunali B, Nicol JM, Burgess LW, Summerell BA. A survey of Fusarium species associated with wheat and grass stem bases in northern Turkey. Sydowia Horn. 2006;58:163-77.

2.Backhouse D, Abubakar AA, Burgess LW, Dennisc JI, Hollaway GJ, Wildermuth GB, et al. Survey of Fusarium species associated with crown rot of wheat and barley in eastern Australia. Australasian Plant Pathology. 2004;33:255-61.

3.Smiley RW, Gourlie JA, Easley SA, Patterson LM, Whittaker RG. Crop Damage Estimates for Crown Rot of Wheat and Barley in the Pacific Northwest. Plant Disease. 2007;89:595-604.

4.Wittwer CT, Herrmann MG, Moss AA, Rasmussen RP. Continuous fluorescence monitoring of rapid cycle DNA amplification. Biotechniques. 2013;54:314-20.

5.Schaad NW, Frederick RD. Real-time PCR and its application for rapid plant disease diagnostics. Canadian Journal of Plant Pathology. 2002. 